\title{
Direct Estimation of the Chemical Potential of Molten NaCl by Molecular Dynamics Simulation
}

\author{
Ryuzo Takagi and Masanori Sakurai \\ Research Laboratory for Nuclear Reactors, Tokyo Institute of Technology, \\ 2-12-1 Ookayama, Meguroku, Tokyo 152, Japan \\ Z. Naturforsch. 53a, 13-16 (1998); received February 11, 1997
}

Molecular dynamics simulation of molten $\mathrm{NaCl}$ was carried out at $1200 \mathrm{~K}$ with well defined potential parameters. The chemical potential of the melt was satisfactorily estimated with the method proposed by Powles et al., which requires only positional data of the ions at the temperature in question, when the number of ions in the basic cell is large enough.

\section{Introduction}

Although the chemical potential can be evaluated by integration of the equation of state, this integration is laborious, since we need to know the pressures from zero density to the density in question [1]. Moreover, on the integration path there may be some phase transitions. However, Widom [2] showed that the canonical ensemble average of $\exp \left(-u_{f} / k T\right)$ gives the excess chemical potential as

$$
\begin{aligned}
\mu^{\mathrm{ex}} & =-R T \ln \left\langle\exp \left(-u_{f} / k T\right)\right\rangle \\
& =-R T \ln \left\{\int_{-\infty}^{\infty} f\left(u_{f}\right) \exp \left(-u_{f} / k T\right) \mathrm{d} u\right\}(1) \\
\beta & =1 / k T,
\end{aligned}
$$

where $R, T, k$, and $f(u)$ are the gas constant, temperature, the Boltzman constant and an energy distribution of $u_{f}$, which is the energy difference between the original system and the system with inserted fictitious particles, respectively.

On the other hand, Shing and Gubbins [3] showed that the canonical ensemble average of $\exp \left(-u_{r} / k T\right)$ gives the excess chemical potential as

$$
\begin{aligned}
\mu^{\mathrm{ex}} & =R T \ln \left\langle\exp \left(u_{r} / k T\right)\right\rangle \\
& =R T \ln \left\{\int_{-\infty}^{\infty} g\left(u_{r}\right) \exp \left(u_{r} / k T\right) \mathrm{d} u\right\},
\end{aligned}
$$

where $g(u)$ is an energy distribution of $u_{r}$, which is the energy difference between the original system and the system with particles removed.

Reprint requests to Dr. R. Takagi; Fax: 81-3-5734-2959.
The chemical potential is given by

$$
\mu=\mu^{\mathrm{ex}}+\mu^{\text {ideal }},
$$

where $\mu^{\text {ideal }}$ is the chemical potential of the ideal gas at the same density and temperature.

Although these two methods require only positional data at the temperature in question, the chemical potentials estimated by these methods depend largely on the lower and upper limits of the energy distributions of $f(u)$ and $g(u)$, respectively. Then Powles et al. [4] showed that the intercept of a linear plot of $L(u)$ vs. $u$ gives the chemical potential, i.e.

$$
L(u) \equiv \ln (f(u) / g(u))=u / k T-\mu^{\mathrm{ex}} / k T,
$$

which means that a plot of $L(u)$ vs. $u / k T$ has a slope of unity and intercept $\beta \mu^{\mathrm{ex}}$.

In this work, in order to show the effectiveness of this method, a molecular dynamics (MD) simulation of molten $\mathrm{NaCl}$, whose potential parameters are well defined, was carried out at $1200 \mathrm{~K}$. Although Larsen, Førland, and Singer [5] successfully estimated the Gibbs energy of molten $\mathrm{NaCl}$, which was obtained by integration over a parameter, the validity of this method has never been demonstrated for ionic melts.

\section{Computational}

In the present study, in order to obtain a canonical ensemble, we made the MD simulation proposed by Nose [6] with the 7 points predictor-corrector method and periodical boundary conditions. The parameter of a heat reservoir ( $q$ in Table 1) [6] was selected carefully. The potential parameters estimated by Tosi and Fumi [7] were employed, and the Coulomb potential was calculated by 
Table 1. Computational results and conditions of the molten $\mathrm{NaCl}$ at $1200 \mathrm{~K}$.

\begin{tabular}{lllll}
\hline$N$ & $\begin{array}{l}q^{*} \\
\left(10^{-43}\right. \\
\left.\mathrm{kg} \mathrm{m}^{2}\right)\end{array}$ & $\begin{array}{l}\text { Internal } \\
\text { energy } \\
(\mathrm{kJ} / \mathrm{mol})\end{array}$ & $\begin{array}{l}\text { Pressure } \\
\left(10^{5} \mathrm{~Pa}\right)\end{array}$ & $\begin{array}{l}\text { Temper- } \\
\text { ature } \\
(\mathrm{K})\end{array}$ \\
\hline 512 & 9.0 & -702.7 & $-9.43 \times 10^{2}$ & 1200.6 \\
1000 & 8.5 & -703.0 & $-7.07 \times 10^{2}$ & 1200.0 \\
1728 & 8.2 & -704.0 & $-7.44 \times 10$ & 1200.0 \\
\hline Experimental & \multicolumn{5}{c}{$-702.2[11]$} \\
\hline
\end{tabular}

* Parameter of a heat reservoir.

the Ewald method. The side lengths of the basic cells, which contain 256, 500 and 864 pairs of $\mathrm{NaCl}$, were estimated with the available density [8]. We chose Gunster and Berendsen's [9] time step of 0.5 fs. After the systems were well annealed, data acquisition was made during 5000 time steps. In every 20 time steps, the positional data were saved in a memory. In order to obtain the energy distributions $g(u)$ in (2), all combinations of a pair of $\mathrm{Na}^{+}$and $\mathrm{Cl}^{-}$(e.g., possible $N \times N$ combinations in the case of the $N$ pairs system) were removed from the systems. On the other hand, in order to estimate $f(u)$ in (1), all combinations of a fictitious particle pair of $\mathrm{Na}^{+}$ and $\mathrm{Cl}^{-}$were inserted into regular $8 \times 8 \times 8,10 \times 10 \times 10$ and $12 \times 12 \times 12$ grids in the basic cells for 256,500 , and 864 pair systems, respectively. Structure factors, pressure and internal energy were also calculated. Although the estimation of pressure embarrassed us, the pressure was successfully evaluated from the transformed virial

$$
\begin{aligned}
\Phi= & E_{\mathrm{c}}+6 E_{\mathrm{dd}}+8 E_{\mathrm{dq}}+\sum_{i, j} \frac{2 \pi b A_{i j} N^{2}}{\sigma V} \\
& \cdot \int_{0}^{\infty} g_{i j}(r) r^{3} \exp \left(\frac{r_{i}^{o}+r_{j}^{o}-r}{\sigma}\right) \mathrm{d} r+K . E .,
\end{aligned}
$$

where $E_{\mathrm{c}}, E_{\mathrm{dd}}, E_{\mathrm{dq}}, V, N, A_{i j}, g_{i j}$ and K. E. are the Coulomb energy, the dipole-dipole interaction energy, the dipole-quadrupole interaction energy, the molar volume, Avogadro's number, the Pauling factor, pair correlation functions, and the kinetic energy respectively, and the other symbols ( $b, \sigma$ and $r_{i}^{0}$ ) are potentials parameters as given by Tosi and Fumi [7]. Equation (5) can be evaluated directly by the virial theorem. For example, the virial $\left(\Phi_{\mathrm{c}}\right)$ of the Coulomb potential $\left(\varphi_{\mathrm{c}}\right)$, which is presented by $E_{\mathrm{c}}$ on the right hand side of (5), is derived as follows:

$$
\begin{aligned}
& \Phi_{\mathrm{c}}=\sum_{i, j} \operatorname{grad} \varphi_{\mathrm{c}} \vec{r}, \\
& \varphi_{\mathrm{c}}=e^{2} z_{i} z_{j} / 4 \pi \varepsilon_{0} r,
\end{aligned}
$$

$$
\begin{aligned}
& \operatorname{grad} \varphi_{\mathrm{c}}=e^{2} z_{i} z_{j} / 4 \pi \varepsilon_{0} r^{3} \vec{r}, \\
& \Phi_{\mathrm{c}}=\sum_{i, j} e^{2} z_{i} z_{j} / 4 \pi \varepsilon_{0} r=E_{\mathrm{c}} .
\end{aligned}
$$

This means that the virial of the Coulomb potential is the Coulomb potential itself.

\section{Results and Discussion}

Partial structure factors, estimated by the Debye equation (6), are shown in Fig. 1 compared with the experimental results obtained by Enderby et al. [10].

$$
S_{i j}(Q)=1+\sum_{i, j} \frac{\sin \left(r_{i j} Q\right)}{r_{i j} Q},
$$

where $Q$ is a wave number and $r_{i j}$ is the separation distance between ions $i$ and $j$. The coincidence between the computational and experimental structure factors is very satisfactory. Temperature, pressure and internal energy, listed in Table 1, seem also reasonable. We concluded that the MD employed in this work simulates the real system very well.

The energy distribution functions $f(u)$ and $g(u)$ are depicted in Figure 2. These functions show a dependence on the number of ion pairs in the basic cell, which may come from the difference of statistical sample number, as shown in Fig. 3, where figures of $\ln (f(u))$, esti-

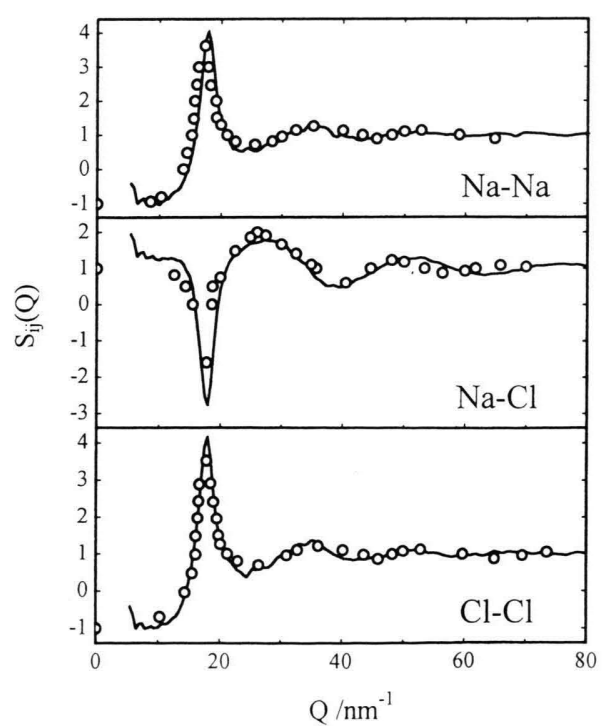

Fig. 1. Partial structure factors of the molten $\mathrm{NaCl}$ solid line: computational and circles: experimental [10]. 


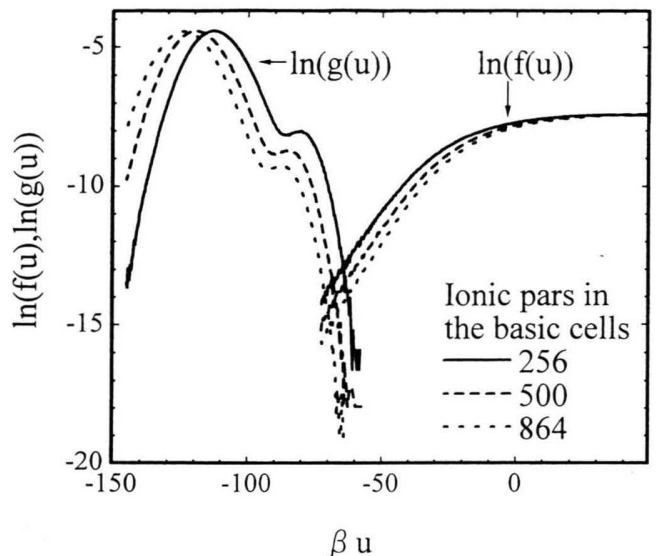

Fig. 2. Energy distribution functions $f(u)$ and $g(u)$, which are described in text.

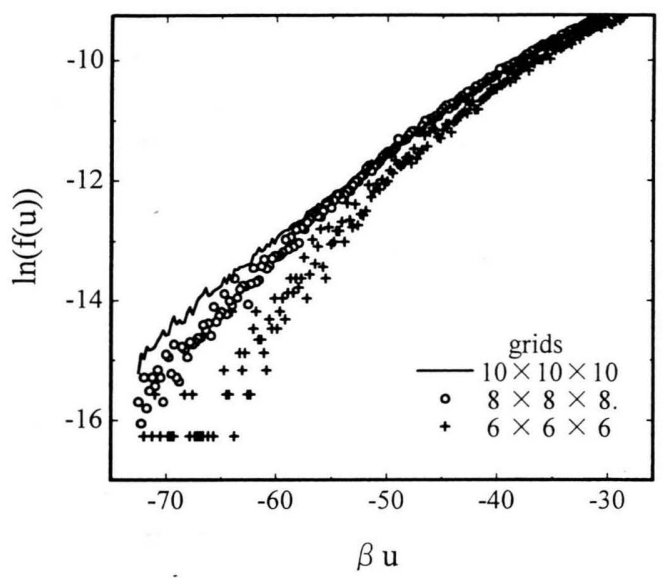

Fig. 3. Variation of $f(u)$ of the 500 pairs system with the grids number.

mated at $6 \times 6 \times 6,8 \times 8 \times 8$ and $10 \times 10 \times 10$ grids for the 500 pairs system, are shown. $L(u)$ defined by (4) is depicted in Fig. 4, where a line expected from the experimental $\mu_{\exp }^{\mathrm{ex}}$ is also shown. The chemical potential of the ideal gas of molten $\mathrm{NaCl}$ at $1200 \mathrm{~K}(-223 \mathrm{~kJ} / \mathrm{mol})$ is calculated by

$$
\begin{aligned}
\mu^{\text {id }} & =k T \log \left(\frac{N}{V} \Lambda_{\mathrm{Na}^{+}}^{3}\right)+k T \log \left(\frac{N}{V} \Lambda_{\mathrm{Cl}^{-}}^{3}\right), \\
\Lambda_{i} & =\sqrt{\frac{h^{2}}{2 \pi m_{i} k T}} \quad\left(i=\mathrm{Na}^{+}, \mathrm{Cl}^{-}\right),
\end{aligned}
$$

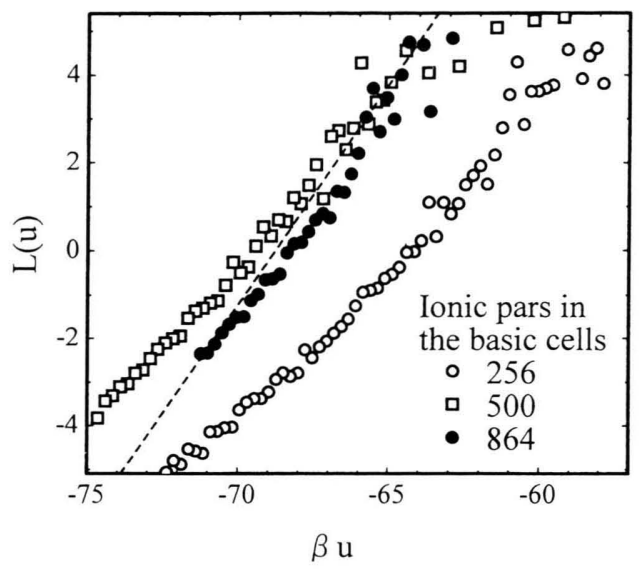

Fig. 4. The function of $L(u)$ defined by Eq. (4). The broken line is the expected line from the experimental results.

Table 2. The excess chemical potentials of the molten $\mathrm{NaCl}$ at $1200 \mathrm{~K}$ estimated by (1), (2), and (4).

\begin{tabular}{rlll}
\hline$N$ & \multicolumn{3}{l}{$\mu^{\mathrm{ex}}(\mathrm{kJ} / \mathrm{mol})$} \\
\cline { 2 - 4 } & $(4)$ & $(2)$ & $(1)$ \\
\hline 512 & -631.7 & -722.4 & -597.7 \\
1000 & -680.4 & -747.2 & -590.1 \\
1728 & -686.8 & -803.2 & -583.81 \\
\hline
\end{tabular}

where $h$ and $m$ are the Plank constant and the mass of an ion, respectively. Figure 4 shows that the system of 256 pairs is not large enough to estimate the chemical potential. The function $L(u)$ of the system for more than 500 pairs locates near the experimental one. We obtained $\mu^{\text {ex }}=-680.4 \mathrm{~kJ} / \mathrm{mol}$ with a slope of 0.91 for the 500 pairs system and $-686.8 \mathrm{~kJ} / \mathrm{mol}$ with a slope of 1.00 for the 864 pairs system, which are very close to the experimental one $(-686.2 \mathrm{~kJ} / \mathrm{mol})$. On the other hand, the chemical potentials estimated by (1) and (2) (Table 2) are far from the experimental one.

\section{Conclusion}

The chemical potential of molten $\mathrm{NaCl}$ was computationally estimated by the method proposed by Powles et al. The function $L(u)$ shows a dependence on the size of the system and gives a reasonable value, if the system contains more than 500 pairs. 
[1] J. P. Valleau and G. M. Torrie, Modern Theoretical Chemistry 5, ed. by J. Berne, Chapt. 5, Plenum (1977).

[2] B. Widom, J. Chem. Phys. 39, 2808 (1963).

[3] K.S. Shing and K. E. Gubbins, Mol. Phys. 46, 1109(1982).

[4] J. G. Powles, W. A. B. Evans, and N. Quirke, Mol. Phys. 46, 1347 (1982)

[5] B. Larsen, T. Førland, and K. Singer, Mol. Phys. 26, 1521 (1973).

[6] S. Nose, Mol. Phys. 52, 255 (1984).
[7] M. P. Tosi and F. G. Fumi, J. Phys. Chem. Solids 25, 45 (1964).

[8] E. R. Van Artsdalen and I. S. Yaffe, J. Phys. Chem. 59, 218 (1955).

[9] W. F. Gunster and H. J. C. Berendsen, Mol. Phys. 34, 1311 (1977).

[10] J. E. Enderby and G. W. Neilson, Adv. Phys. 29, 323 (1980)

[11] M. P. Tosi, Solid State Phys. 16, 1 (1964). 